\title{
Die Struktur des Ratanhins
}

von

\section{Guido Goldschmiedt,}

w. M. k. Akad.

Aus dem II. chemischen Universitätslaboratorium in Wien.

(Vorgelegt in der Sitzung am 18. Februar 1913.)

Vor einigen Monaten habe ich eine Reihe von Versuchen mitgeteilt, ${ }^{1}$ welche ich mit einem kleinen Quantum des im Titel genannten Stoffes ausführen konnte; hierdurch ist die Struktur des Ratanhins nicht vollständig aufgeklärt worden und ich habe am Schlusse meiner Abhandlung die Absicht ausgesprochen, da das Material, das ich besaß, erschöpft war, durch synthetische Versuche die noch fraglichen Punkte aufzuklären.

In der 'Tat habe ich' solche Untersuchungen in Gemeinschaft mit Dr. A. Obermayer unternommen und wir haben zunächst die Synthese des - $\gamma$-p-Oxyphenylpropylamins $\mathrm{HO} \cdot \mathrm{C}_{6} \mathrm{H}_{4} \cdot \mathrm{CH}_{2} \cdot \mathrm{CH}_{2} \cdot \mathrm{CH}_{2} \cdot \mathrm{NH}_{2}$ durchgeführt, welche mit der aus Ratanhin durch Kohlendioxydabspaltung erhaltenen Base verglichen werden sollte, worüber demnächst berichtet werden soll.

Daß ich die Synthese gerade dieser Base zuerst in Angriff genommen habe, beruht darauf, daß ich es wegen der großen Ähnlichkeit des Ratanhins mit dem Tyrosin für wahrscheinlich hielt, daß ersteres wie dieses ein primäres Amin sei.

\footnotetext{
1 M., 33, 1379 (1912).
} 
Inzwischen hat mir Herr Prof. Hönigschmid eine kleine Quantität (etwa $2 \mathrm{~g}$ ) von W. Gintl sen. stammendes Ratanhin überlassen, das sich in der Sammlung seines Institutes befand. Es sei ihm auch an dieser Stelle dafür gedankt, daß er es mir hierdurch möglich gemacht hat, dem früher Ermittelten noch einige, für die Erkenntnis der Struktur des Ratanhins wertvolle und ausschlaggebende Ergänzungen hinzuzufügen.

Zunächst wurde untersucht, ob Ratanhin tatsächlich eine primäre Base ist; die nach Herzig-Meyer ausgeführte Bestimmung des Methyls am Stickstoff lieferte ein positives Ergebnis.

$0 \cdot 2872 g$ Substanz lieferten $0 \cdot 3108 g$ Jodsilber.

In 100 Teilen:

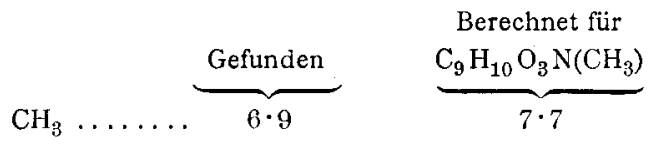

Hieraus ergibt sich mit Sicherheit, daß Ratanhin die Struktur einer $p$-Oxyphenylmethylaminopropionsäure besitzt und da $B$ nur noch in Frage kommen könnte, ob es Methyltyrosin ( $\beta, p$-Oxyphenyl- $\alpha$-methylaminopropionsäure) oder $\beta, p$-Oxyphenyl- $\beta$-methylaminopropionsäure ist.

Methyltyrosin ist vor einigen Jahren von Fri edmann und Gutman $n^{1}$ synthetisch dargestellt worden und in allerjüngster Zeit haben Treat B. Johnson und Ben H. Nicolet ${ }^{2}$ dasselbe noch auf einem anderen Wege bereitet. Herr Prof. Johnson hatte die Liebenswürdigkeit, mir einen Separatabdruck seiner Arbeit zu übersenden, so daß ein Vergleich der Eigenschafter des Ratanhins und jener des Methyltyrosins möglich ist. Übrigens habe ich Herrn Prof. Johnson auf seinen Wunsch den letzten kleinen Rest meines Vorrates an Ratanhin übersendet, so daß die unmittelbare Vergleichung der beiden Präparate in Aussicht steht.

Die Schmelzpunkte, richtiger Zersetzungspunkte, können allein zur Identifizierung nicht herangezogen werden, weil sie

1 Biochem. Zeitschr., 27, 496 (1910).

2 American. chem. Journ., 47, Ni. 6 (1912). 
je nach Art des Erhitzens innerhalb recht weiter Grenzen schwanken; so geben Friedmann und Gutmann von dem Methyltyrosin an:

Zersetzungspunkt: »scharf bei $318^{\circ}$ (unkorr.) .

Johnson-Nicolet: "anywhere between $265^{\circ}$ and $320^{\circ}$ according to the rate of heating «.

Goldschmiedt für Ratanhin: "bei raschem Erwärmen ist Sinterung bei $250^{\circ}$, bei $265^{\circ}$ Beginn der Verflüssigung zu beobachten, sie ist bei zirka $280^{\circ}$ beendet. «

Der Vorschrift von Abderhalden und Guggenheim ${ }^{1}$ folgend, welche sich für die Darstellung der aus Gorgonin und aus Spongin durch Hydrolyse gewonnene Jodgorgosäure (3,5-Dijodtyrosin) aus Tyrosin bewährt hatte, habe ich die Gewinnung eines Dijodratanhins durch Einwirkung von Jod auf alkalische 1-Ratanhinlösung versucht. Die analoge Reaktion haben Johnson und Nicolet ${ }^{2}$ mit ihrem synthetischen, also d-1-Methyltyrosin durchgeführt; da nach Beobachtungen von Wheeler und Jamieson ${ }^{3}$ das Tyrosin sich bei dieser Behandlung racemisiert, ist man wohl berechtigt, bei dem Ratanhin dasselbe $z u$ erwarten, so daß die beiden Präparate identisch sein dürften, wenn die Ausgangsmaterialien dieselbe Struktur besitzen.

Die Erscheinungen bei der Jodierung entsprechen genau jenen, welche Johnson und Nicolet bei ihrem Präparate beobachten konnten, sie sind auch jenen, die beim Tyrosin auftreten, durchaus ähnlich. Das Rohprodukt ist ziemlich dunkel gefärbt und wurde durch Behandlung mit Tierkohle in sehr verdünnt ammoniakalischer Lösung entfärbt, durch verdünnte Essigsäure wurden kaum gefärbte, mikroskopische Nädelchen ausgefällt.

Der Zersetzungspunkt des Präparates lag bei $206^{\circ}$, bei einer anderen Bestimmung bei $207^{\circ}$, während Johnson und Nicolet aus dem von ihnen aus Methyltyrosin erhaltenen

\footnotetext{
I Berl. Ber., 41, 1, 1238 (1908).

${ }^{2}$ L. c.

3 Abderhalden, Biochem. Handlexikon, IV, 706, aus: Americ. Chem. Journ., 33, 365 (1905).
} 
Dijodderivate, je nach der Geschwindigkeit des Erhitzens, Verflüssigung $z$ wischen 200 bis $210^{\circ}$ beobachteten.

6.32 mg Substanz, bei $109^{\circ}$ getrocknet, lieferten bei Anwendung des F. Preglschen Mikroverfahrens 6, $54 m g$ Jodsilber. 1

In 100 Teilen:

$$
\mathrm{J} \ldots \ldots \ldots \ldots \underbrace{\text { Gefunden }}_{55 \cdot 94} \quad \underbrace{\begin{array}{c}
\text { Berechnet für } \\
\mathrm{C}_{10} \mathrm{H}_{11} \mathrm{~J}_{2} \mathrm{NO}_{3}
\end{array}}_{56 \cdot 7}
$$

Es liegt hiernach Dijodratanhin vor.

Ist Ratanhin identisch mit Methyltyrosin ( $\beta$ - $p$-Oxyphenyl$\alpha$-methylaminopropionsäure), wie nach Obigem angenommen werden muß, dann muß auch die durch Kohlendioxydabspaltung daraus von mir erhaltene Base ${ }^{2}$ identisch sein mit $\beta$ - $p$-Oxyphenyläthylmethylamin, einer Substanz, die bereits von George Stanley Walpole ${ }^{3}$ synthetisch bereitet worden ist. Ich habe daher die Base aus Ratanhin, welche ich früher nicht im reinen Zustande darstellen konnte - nur das salzsaure Salz war beschrieben worden - mit dem letzten Reste meines Ratanhins in der bereits beschriebenen Weise nochmals bereitet; das Destillat von Ratanhin wurde nach einiger Zeit krystallinisch; es wurde aus Toluol, dann zweimal aus Benzol umkrystallisiert. Die so erhaltenen nahezu farblosen, zu Drusen vereinigten Blättchen verflüssigten sich dann bei 128 bis $129^{\circ}$. Die Substanz ist in Wasser schwer löslich, sie zeigt Millon's Reaktion; die wässerige Lösung reagiert stark alkalisch.

$5.39 \mathrm{mg}$ Substanz, bei $109^{\circ}$ getrocknet, lieferten, nach F. Pregl verbrannt, $14 \cdot 19 \mathrm{mg}$ Kohlendioxyd und $4 \cdot 35 \mathrm{mg}$ Wasser.

In 100 Teilen:

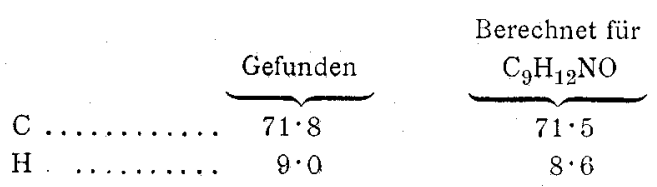

1 Die Ausfübrung dieser sowie der folgenden Mikroanalyse verdanke ich meinem Assistenten Herrn Dr. Ernst Philippi.

2 L. c.

3 Journ. of the chem. Soc. Transactions, 97, 1, 945 (1910). 
Eine kleine Quantität wurde in das Chlorhydrat umgewandelt, dasselbe wurde bei $109^{\circ}$ getrocknet, in wenig absolutem Alkohol, in welchem es sehr leicht löslich ist, aufgenommen, dann mit trockenem Äther gefällt und diese Operation wiederholt. Die so erhaltenen zarten Nädelchen schmolzen bei 146 bis $147^{\circ}$.

Eine kleine Quantität des Chlorhydrates wurde auf einem Uhrgläschen in einigen Tropfen Wasser gelöst, ein Tropfen verdünnte Salzsäure und ein Tropfen Platinchlorwasserstoffsäurelösung zugesetzt; bald schieden sich gelbe Kryställehen aus, die auf einer Tonplatte trocken gesaugt und auf derselben zweimal mit eiskaltem Wasser gewaschen wurden; der Schmelzpunkt lag dann bei 205 bis $206^{\circ}$ (korr.).

Herr Prof. F. Emich hatte die Liebenswürdigkeit, wofür ihm auch an dieser Stelle gedankt sei, das Chloroplatinat nach seinen ausgezeichneten Mikroverfahren zu analysieren.

Die Bestimmungen sind mit zwei verschiedenen Mikrowagen ausgeführt worden, bei welchen der Wert eines Teilstriches $0.011 \mathrm{mg}$, beziehungsweise $0.0057 \mathrm{mg}$ betrug.

I. 48.47 Teilstriche Substanz (zirka $0.5 \mathrm{mg}$ ), bei $103^{\circ}$ getrocknet, gaben $13 \cdot 22$ Teilstriche Platin.

II. 57.95 Teilstriche Substanz (zirka $0.6 \mathrm{mg}$ ) gaben 15:80 Teilstriche Platin.

III. $95 \cdot 88$ Teilstriche Substanz (zirka $0.55 \mathrm{mg}$ ) gaben $26 \cdot 18$ Teilstriche Platin. In 100 Teilen:

$\overbrace{\begin{array}{ccc}\text { I } & \text { II } & \text { III } \\ 27 \cdot 3 & 27 \cdot 3 & 27 \cdot 3\end{array}}^{\text {Gefunden }} \quad \underbrace{\left(\mathrm{C}_{9} \mathrm{H}_{13} \mathrm{NO}\right)_{2} \cdot \mathrm{H}_{2} \mathrm{PtCl}_{6}}_{27 \cdot 4}$

Nachstehend die vergleichende Zusammenstellung meiner Beobachtungen an dem Spaltungsprodukte des Ratanhins mit jenen, welche Walpole an dem $\alpha$-p-Oxyphenyläthylmethylamin gemacht hat:

$$
\text { Goldschmiedt Walpole }
$$

Zersetzungspunkt der

$$
\text { Base........... Scharf } 130^{\circ}
$$

Zersetzungspunkt des

Chlorhydrats ....146 bis $147^{\circ} \quad 148 \cdot 5^{\circ}$

Zersetzungspunkt des

Chlorplatinats ...205 bis $6^{\circ}$ (korr.) $205^{\circ}$ (korr.) 
Dic Übereinstimmung ist eine ausreichende, um behaupten zu können, daß die beiden Basen identisch sind, und damit ist der Beweis erbracht, daß Ratanhin die Struktur der $\beta, p$-Oxyphenyl- $\alpha$-methylaminopropionsäure besitzt.

Durch die Publikation von Johnson und Nicolet bin ich auf eine Arbeit von Hiller-Bombien: ${ }^{1}$ »Beiträge zur Kenntnis der Geoffroya-Rinden « aufmerksam gemacht worden; sie ist pharmakognostischen und chemischen Inhalts. In dem chemischen Teile der Abhandlung, welcher die Literatur des Gegenstandes wohl vollständig berücksichtigt, wird gezeigt, daß das schon 1824 von Hüttenschmid in Geoffraya-Rinden aufgefundene, aber nicht analysierte Geoffroyin dieselbe Zusammensetzung hat wie Ratanhin und daß es demselben so ähnlich sei, daß Hiller-Bombien die beiden Substanzen für identisch hält.

Dieselbe Substanz ist auch noch in anderen Papilionaceen aufgefunden worden und erscheint unter dem Namen Surinamin,? Geoffroyin, Angelin, Ratanhin und Andirin in der Literatur.

Diese Bezeichnungen sind demnach Synonima für den rationellen Namen $\beta$ - $p$-O x y phen y l- $\alpha$-met hyla minopropionsäure, dem die Formel

$$
p-\mathrm{HO} \cdot \mathrm{C}_{6} \mathrm{H}_{4} \cdot \mathrm{CH}_{2} \cdot \mathrm{CH}\left(\mathrm{N}_{\mathrm{H}}^{\mathrm{CH}_{3}}\right) \cdot \mathrm{COOH}
$$

entspricht.

1 Arch. d. Pharmazie, 230, 513. (Die Arbeit enthält die einschlägige Literatur.)

2 H. Blau (Zeitschr. für physiol. Chemie, 58,153 [1908] hat Surinamin aus Geoffraya-Rinde dargestellt und gezeigt, daß bei der trockenen Destillation eine Base entsteht, deren Chloroplatinat bei $205^{\circ}$ schmilzt, wie er jetzt auch bei den analogen Substanzen aus Ratanhin und aus synthetischem Methyltyrosin gefunden worden ist. Bei der Kalischmelze des Surinamins konstatierte er die Bildung von $p$-Oxybenzoesäure. 